\title{
Exon deletions of the phenylalanine hydroxylase gene in Italian hyperphenylalaninemics
}

Francesco Calì ${ }^{1}$, Giuseppa Ruggeri ${ }^{1}$, Mirella Vinci ${ }^{1,6}$, Concetta Meli ${ }^{2}$, Carla Carducci ${ }^{3}$, Vincenzo Leuzzi ${ }^{4}$, Simone Pozzessere ${ }^{4}$, Pietro Schinocca ${ }^{1}$, Alda Ragalmuto ${ }^{1}$, Valeria Chiavetta ${ }^{1}$, Salvatore Micciche ${ }^{5}$ and Valentino Romano $0^{1,6,7}$

${ }^{1}$ Laboratorio di Genetica Molecolare

Associazione Oasi Maria SS. (I.R.C.C.S.)

Troina (EN), Italy

${ }^{2}$ Centro Malattie Metaboliche e Congenite

Azienda Ospedaliera Universitaria

Policlinico di Catania

Catania, Italy

${ }^{3}$ Dipartimento di Medicina Sperimentale

${ }^{4}$ Dipartimento di Scienze Neuropsichiatriche dell'età evolutiva Università La Sapienza

Roma, Italy

${ }^{5}$ Dipartimento di Fisica e Tecnologie Relative

${ }^{6}$ Dipartimento di Oncologia Sperimentale e Applicazioni Cliniche Università degli studi di Palermo

Palermo, Italy

${ }^{7}$ Corresponding author: Tel, 39-91-6722852;

Fax, 39-91-6806420; E-mail, vromano @ unipa.it

This paper is dedicated to the memory of Carmelita Risicato.

DOI 10.3858/emm.2010.42.2.009

Accepted 6 November 2009

Available Online 30 November 2009

Abbreviations: CMDA, comparative multiplex dosage analysis; DGGE, denaturing gradient gel electrophoresis; DHPLC, denaturing high performance liquid chromatography; MHP, mild hyperphenylalaninemia; MLPA, multiplex ligation-dependent probe amplification; $\mathrm{PAH}$, phenylalanine hydroxylase; RPA, relative peak area

\footnotetext{
Abstract

A consistent finding of many studies describing the spectrum of mutant phenylalanine hydroxylase (PAH) alleles underlying hyperphenylalaninemia is the impossibility of achieving a $100 \%$ mutation ascertainment rate using conventional gene-scanning methods. These methods include denaturing gradient gel electrophoresis (DGGE), denaturing high performance liquid chromatography (DHPLC), and direct sequencing. In recent years, it has been shown that a significant pro-
}

portion of undetermined alleles consist of large deletions overlapping one or more exons. These deletions have been difficult to detect in compound heterozygotes using gene-scanning methods due to a masking effect of the non-deleted allele. To date, no systematic search has been carried out for such exon deletions in Italian patients with phenylketonuria or mild hyperphenylalaninemia. We used multiplex ligation-dependent probe amplification (MLPA), comparative multiplex dosage analysis (CMDA), and real-time PCR to search for both large deletions and duplications of the phenylalanine hydroxylase gene in Italian hyperphenylalaninemia patients. Four deletions removing different phenylalanine hydroxylase (PAH) gene exons were identified in 12 patients. Two of these deletions involving exons 4-5-6-7-8 (systematic name c.353-?_912 + ?del) and exon 6 (systematic name c.510-?_706 + ?del) have not been reported previously. In this study, we show that exon deletion of the PAH gene accounts for $1.7 \%$ of all mutant PAH alleles in Italian hyperphenylalaninemics.

Keywords: gene deletion; gene dosage; ligase chain reaction; phenylalanine hydroxylase; phenylketonurias

\section{Introduction}

Phenylketonuria (MIM 261600) is the most common inherited disorder of amino acid metabolism. It is transmitted by an autosomal recessive mode of inheritance caused by mutations of the phenylalanine hydroxylase (PAH) gene (Scriver and Kaufman, 2001). Genotyping of patients with PAH deficiency is, in most cases, useful to reach a differential diagnosis as early as possible after birth (Guldberg et al., 1998), thus improving the efficacy of dietary treatment. In addition, mutation analysis is useful to identify a subgroup of tetrahydrobiopterin-responsive patients with $\mathrm{PAH}$ deficiency (Belanger-Quintana et al., 2005) and for prenatal diagnosis (Romano et al., 1994).

The identification of PAH mutant alleles in a newly-diagnosed patient is complicated by the large number of mutations underlying the disease. To date, more than 500 different PAH gene mutations have been identified with nearly $90 \%$ of them being point mutations (http://www.pahdb. mcgill.ca/). This strong degree of molecular 
A

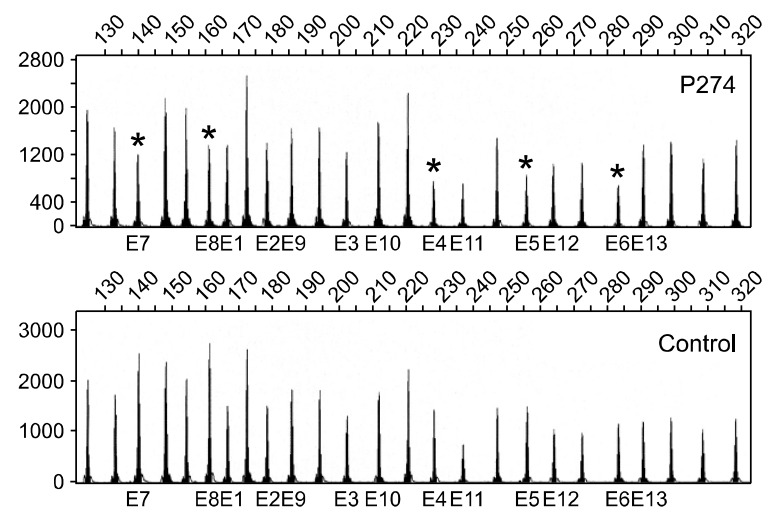

B

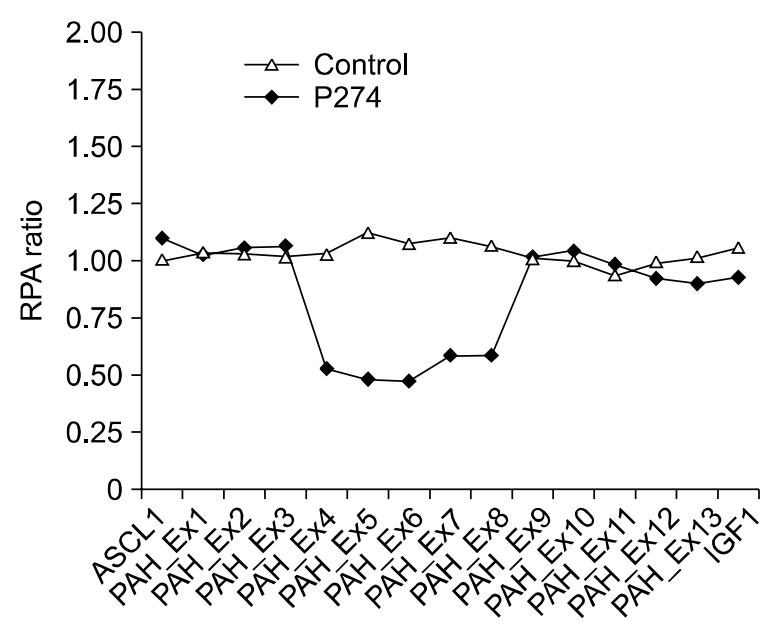

Figure 1. MLPA analysis performed on patient P274 with a deletion of the PAH gene removing 5 exons (c.353 - ?_912 + ?del). (A) In the electropherogram the asterisks above peaks indicate the deleted exons (E4, E5, E6, E7, E8) in the patient DNA, compared to control DNA. (B) Graph displaying ratios of patient P274 and control relative peak areas (RPA) determined for each exon of the PAH gene. Reference values are as follows: RPA Ratio - $1=$ normal dosage; RPA Ratio - 0.5 = Heterozygous deletion. IGF1 and ASCL1 control probes used with the MLPA kit corresponding to two genes flanking the $\mathrm{PAH}$ gene. Peaks lacking the indication of the PAH gene exons refer to other control probes included in the kit.

heterogeneity has motivated development of a variety of PCR-based gene-scanning approaches that have allowed a high identification rate for $\mathrm{PAH}$ gene mutations in many populations (http://www. pahdb.mcgill.ca). Nevertheless, in many of these studies, the mutant genotype has remained partially or completely undetermined in about less than $10 \%$ of patients (Mirisola et al., 2001). In recent years, several studies (Gable et al., 2003; Desviat et al., 2006; Kozak et al., 2006; Birk Moller et al., 2007; Lee et al., 2008) have shown that a significant proportion of these undetermined alleles consist of large deletions overlapping one or more exons. These deletions have been difficult to detect in compound heterozygotes using conventional gene-scanning methods (e.g. DGGE, DHPLC) due to a masking effect of the non-deleted allele. To date, no systematic search for exon deletions or duplications of the PAH gene has been carried out in Italian phenylketonuria and mild hyperphenylalaninemia (MHP) patients.

We used multiplex ligation-dependent probe amplification (MLPA) (Schouten et al., 2002) to screen for deletions/duplications of $\mathrm{PAH}$ gene exons in 43 Italian hyperphenylalaninemics whose genotypes remained partially or completely undetermined following denaturing gradient gel electrophoresis (DGGE) and DNA sequencing analyses. Several deletions, including two new ones, were identified in 12 patients. All deletions were also confirmed using comparative multiplex dosage analysis (CMDA) and real-time PCR.

\section{Results and Discussion}

According to MLPA analysis, one duplication and six deletions of several exons of the PAH gene were detected in 13 out of 43 tested patients. These mutations included, (i) a deletion of exon 3 in eight patients (F2, F115, P469, P259, P446, P291 P657, P735), (ii) a deletion of exon 5 in two patients (F48, F118), (iii) a large deletion of exons 4-5-6-7-8 in one patient (P274) (see Figure 1), (iv) a deletion of exons 6-7 in one patient (P628), and (v) a duplication of exon 11 in one patient (F81). However, when the latter patients (F81 and P628) were retested using real-time- PCR and CMDA, gene dosage variation was not confirmed for both the duplication of exon 11 (F81) and the deletion of exon 7 (P628) (see Figures 2 and 3). In addition, Figure 3 shows the results of gene dosage analyses performed using real-time PCR for all deleted exons. The deletion detected using MLPA in patient $\mathrm{P} 628$ is likely an artefact caused by a point mutation (c.754C- $>\mathrm{T}$ ) in a region of exon 7 that overlapped the MLPA sequence probe used to analyze the exon. On the other hand, one possible explanation accounting for the inconsistent result obtained in patient F81 (apparent duplication of exon 11) with different gene dosage methods could be the hybridization instability of MLPA probes. Indeed, Zheng et al. (2008), using array-MLPA, found that the same longer probes (142-355 bp) used in the MLPA kit from Hollanddisplay hybridization instability, compared to smaller probes. 
A

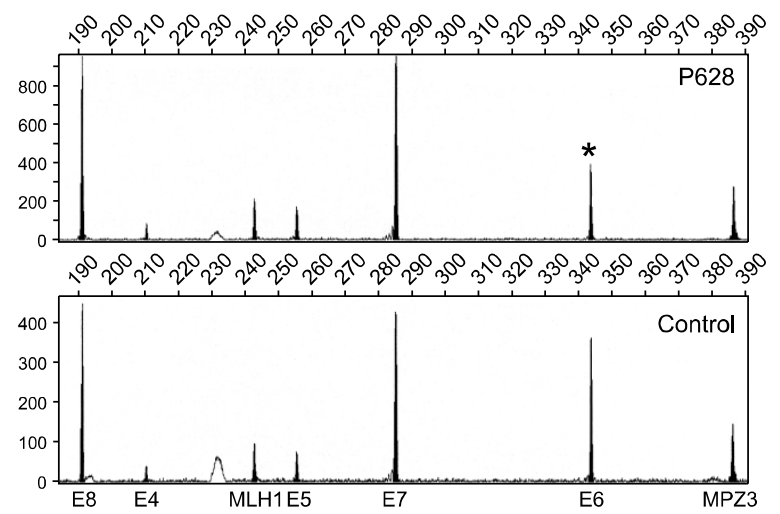

B

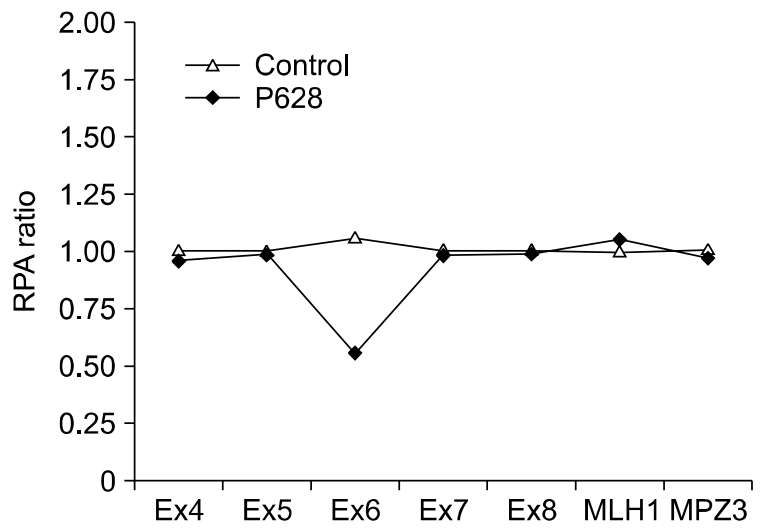

Figure 2. CMDA analysis performed on patient P628 with a deletion of the PAH gene removing exon 6 (c.510 - ? $706+$ ?del). (A) In the electropherogram, the asterisk above the peak indicates the deleted exon (E6) in the patient DNA compared to control DNA. (B) Graph displaying ratios of patient P628 and control relative peak areas (RPA) determined for exons of the PAH gene. Reference values are as follows: RPA Ratio - $1=$ normal dosage; RPA Ratio - $0.5=$ Heterozygous deletion. MLH1 and MYE are control probes used in the CMDA analysis.

A summary of all exon deletions detected in 12 of 43 patients confirmed using the three gene dosage methods is shown in Table 1, which also shows information on, (i) the second mutant allele (if available), (ii) the intragenic STR-VNTR minihaplotypes associated with each mutation (when available), and (iii) the clinical phenotype of the patients. The deletion removing exons 4-5-6-7-8 (c.353 - ?_912 + ?del) (pat. \# P274) and the deletion of exon 6 (c.510 - ?_706 + ?del) (pat. \# P628) have not been reported previously.

Since phenylketonuria deletions of exons 3 and

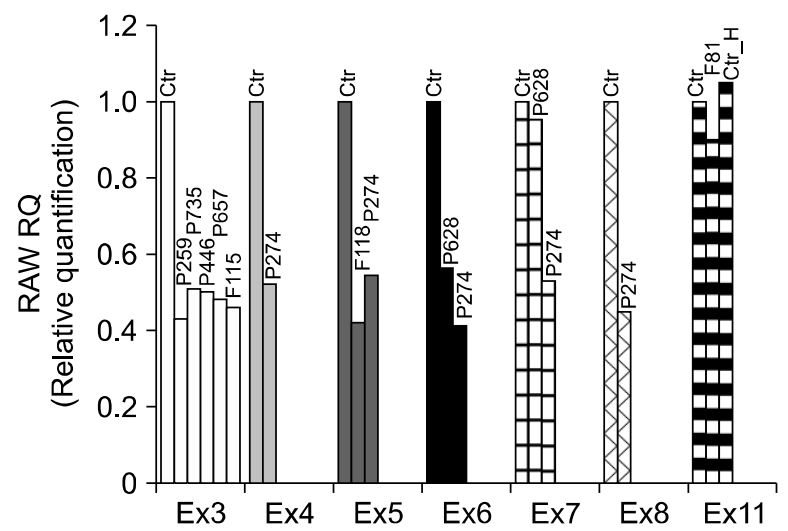

Figure 3. Real-time PCR results for the PAH gene in hyperphenylalaninemia patients with exon deletions. The histograms show the mean relative quantification $(R Q)$ values computed only for exons shown as positive in MLPA analysis. Each sample was run in triplicate and each experiment was confirmed at least twice. Note that patient F81, positive in MLPA for a duplication of exon 11, displays a normal dosage when retested using real-time PCR. ctr, a normal sample used as a control; EX, exon; $\mathrm{Ctr}_{\mathrm{H}} \mathrm{H}$, control DNA from a heterozygote for one point mutation in exon 11.
5 have also been described in other patients (Desviat et al., 2006; Kozak et al., 2006) we attempted to verify the same PAH gene breakpoints for these deletions in our patients. Using PCR and sequence analysis we identified, in three out of 10 cases (patients F2, P735, P657), the same breakpoints removing the 4765 nucleotides of exon 3 (g.21560_26324del4765) as detected by Kozak et al. (2006). Also, the deletion of exon 5 identified in our patient F118 (Figure 4) had the same breakpoints identified in patients reported by Kozak et al. (2006) (g.50448_51402del955). Deletions of exons 3 and 5 detected in our F2 and F118 patients, respectively, and in the patients studied by Kozak et al. (2006), also shared the same intragenic VNTR alleles (VNTR allele \# 8 exon 3 deletion and VNTR allele \# 7 -exon 5 deletion; Table 1). These conserved intragenic allele associations suggest a common origin for these two deletions detected in both Czech and Sicilian populations. To date, several different breakpoints removing either exon 3 or 5 have been described. As indicated by Kozak et al. (2006) these findings support the hypothesis of the existence of recombination hot spots within the $\mathrm{PAH}$ gene. The undetermined allele in patient F118 and the 62 alleles that have shown negative results for a mutation search performed using DGGE, DHPLC, DNA sequencing, and MLPA, indicate the possibility that another type of mutation (other than exon deletions, duplications, or a point mutation in exons or exon/intron junctions) can inactivate $\mathrm{PAH}$ gene function. Such other type of mutation could be, for example, either an intronic mutation regulating mRNA splicing or a mutation in the PAH gene promoter. 
Table 1. Deletions and duplications of the PAH gene detected in Italian hyperphenylalaninemics.

\begin{tabular}{llllll}
\hline Fam \# & \multicolumn{1}{c}{ Mutation 1* } & STR-VNTR & \multicolumn{1}{c}{ Mutation 2* } & STR-VNTR & Phenotype \\
\hline F2 & g.21560_26324del4765* & $8-244$ & c.1066-11G- $>$ A & $7-252$ & Classic PKU \\
F48 & c.442-?_509 + ?del & $3-244$ & c.442-?_509 + ?del & $3-244$ & Mild PKU \\
F115 & c.169-?_352 + ?del & $7-244$ & c.782G- $>$ A & $8-240$ & Mild PKU \\
F118 & g.50448_51402del955** & $7-244$ & n.d. & $8-240$ & Mild PKU \\
P274 & c.353-?_912 + ?del & $3-240$ & c.331C- $>$ T & $3-236$ & Classic PKU \\
P469 & c.169-?_352 + ?del & n.d. & c.782G- $>$ A & n.d. & Classic PKU \\
P259 & c.169-?_352 + ?del & $7-244$ & c.1066-11G- $>$ A & $7-252$ & Classic PKU \\
P446 & c.169-?_352 + ?del & $7-244$ & c.143T- $>$ C & $3-236$ & Classic PKU \\
P291 & c.169-?352 + ?del & $7-244$ & c.782G- $>$ A & $8-240$ & Classic PKU \\
P735 & g.21560_26324del4765** & n.d. & c.782G- $>$ A & n.d. & Classic PKU \\
P628 & c.510-?_706 + ?del & n.d. & c.754C- $>$ T & n.d. & Classic PKU \\
P657 & g.21560_26324del4765** & n.d. & c.842C- $>$ T & n.d. & Classic PKU \\
\hline
\end{tabular}

*, Systematic name; ${ }^{*}$, These deletions have the same breakpoint detected by Kozak et al. (2006) in Czech hyperphenylalaninemia patients (see text for explanation). PKU, phenylketonuria; n.d., not determined.

In summary, exon deletion of the PAH gene accounts for $1.7 \%(13 / 759)$ of all mutant PAH alleles in Italian phenylketonuria and non-phenylketonuria-hyperphenylalaninemia patients, corresponding to an overall mutation detection rate of $95.6 \%$. We have shown that MLPA is useful for detection of exon deletions of the PAH that would otherwise go undetected in compound heterozygotes using conventional gene-scanning methods. However, there are limitations in the performance of MLPA for quantification analysis, possibly leading to false positives. The use of other gene dosage analysis methods is indispensable to confirm preliminary MLPA findings and avoid false positive results when genotyping hyperphenylalaninemia patients.

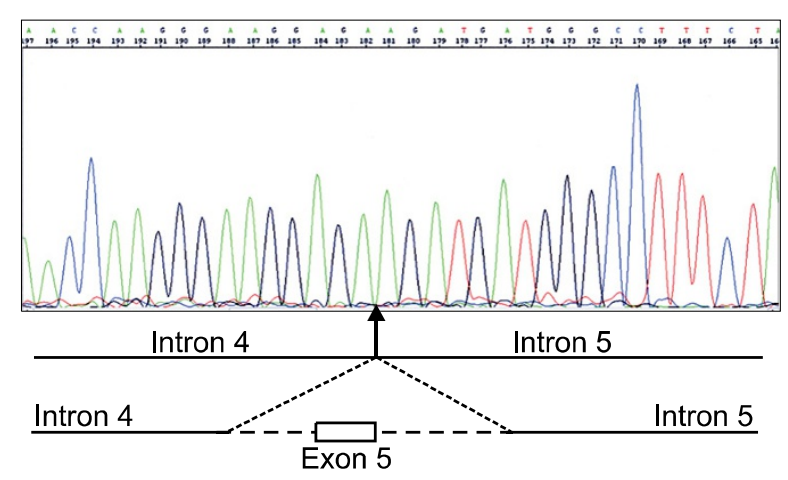

Figure 4. Breakpoint sequence analysis of the deletion ( 9.50448 51402del955) detected in patient F118 removing exon 5 and parts of the flanking introns 4 and 5 of the PAH gene. Sequence analysis revealed that this deletion is identical to the deletion reported by Kozak et al. (2006) in two Czech phenylketonuria patients of Slavic origin.

\section{Methods}

\section{Patients}

We used MLPA to test 43 patients diagnosed with hyperphenylalaninemia at the Center for the Study of Inherited Metabolic Diseases, University of Catania (15 patients) and at the Department of Neuropsychiatric Sciences of Developmental Disorders, University of Rome "La Sapienza" (28 patients). Informed consent was obtained from patient's parents and the research was been approved by the Ethical Committee of the OASI Institute. Clinical and metabolic phenotypes were assessed according to the criteria adopted by Guldberg et al. (1998). DNA samples for the patients were already available in the DNA bank of hyperphenylalaninemia patients located at the OASI Institute (Troina). The 43 patients were selected for $\mathrm{PAH}$ gene exon deletion screening because either one or both PAH mutant alleles remained unknown following previous DGGE/DHPLC and DNA sequencing analyses performed on a total of 802 alleles (Mirisola et al., 2001), [F. Calì and C. Carducci unpublished data].

\section{MLPA analysis}

MLPA analysis was performed using the Salsa MLPA Kit P055 PAH (MRC-Holland, Amsterdam, The Netherlands). MLPA analysis was carried out as described by Schouten et al. (2002). PCR products were identified and quantified using capillary electrophoresis on an $\mathrm{ABI} 3130$ genetic analyser with the Gene Mapper software from Applied Biosystems (Foster City, CA).

In order to efficiently process MLPA data, a spreadsheet was generated in Microsoft Excel. First, data corresponding to each sample (patient and control DNA) were normalised by dividing each probe signal strength value (the area of each peak) by the average signal strength of 10 control probes to generate a relative peak area (RPA) for each peak. The RPA for each probe in a patient sample was then compared to the RPA for a control sample by dividing the patient RPA by the control RPA for each peak. This value was then used to define the following categories 
according to Akrami et al. 2005: (i) "normal" for values in the range $0.75-1.25$ (these values correspond to more than three standard deviations above and below the mean of the normalized data); (ii) "deleted" for values smaller than 0.60 , and (iii) "duplicated" for values larger than 1.40 (these values correspond to more than five standard deviations above and below the mean of the normalized data).

As a quality test for the probes, we computed the standard deviation (SD) of the normalised signals for the ten control probes for both patients and control individuals. MLPA analysis was repeated for samples yielding an SD value exceeding the threshold value of $\sigma_{t}=0.05$. This value corresponds approximately to the mean value of the standard deviations of the normalized data relative to the ten control probes for all individuals (both patients and control). Patients showing positive in MLPA were then retested using real-time PCR and comparative multiplex dosage analysis (CMDA) to confirm the results of the MLPA analysis.

\section{Real-time PCR}

Relative quantification was performed using a 7500 RealTime PCR System (Applied Biosystems) with SYBR green dye chemistry (Applied Biosystems). The primers used (sequences available on request) allowed amplification of each exon and the exon/intron junctions of the PAH gene. Each reaction $(50 \mu \mathrm{l})$ used $25 \mu \mathrm{l}$ of SYBR Green Master Mix, $0.5 \mu \mathrm{l}[5 \mu \mathrm{M}]$ of forward and reverse primers, $5 \mu \mathrm{l}$ of [50 ng] DNA, and $19 \mu \mathrm{l}$ of $\mathrm{H}_{2} \mathrm{O}$.

GPR15 (Genebank: NT_005612) was used as reference gene to normalize differences between samples and was simultaneously analyzed in a separate tube for each assay. Control DNAs with a known disomic genotype for the relevant exons were used from healthy subjects, heterozygotes, or compound heterozygous phenylketonuria patients. The real-time PCR cycling conditions were 2 ' min at $50^{\circ} \mathrm{C}$, followed by $10 \mathrm{~min}$ at $95^{\circ} \mathrm{C}, 40$ cycles at $95^{\circ} \mathrm{C}$ for $15 \mathrm{~s}$, and $60^{\circ} \mathrm{C}$ for $60 \mathrm{~s}$. Melting curve analysis was performed to check the accuracy of every PCR amplification. Each assay included a no-template control. Each sample was run in triplicate. PAH gene exon deletions were determined using the comparative threshold cycle method $(\Delta \Delta \mathrm{Ct}$ ) with the SDS V1.2.1 software (Applied Biosystems).

\section{Comparative multiplex dosage analysis (CMDA)}

CMDA was performed as described by Gable et al. (2003) with some modification to confirm the deletions detected using MLPA in the 12 patients. In brief, all exons involved in a deletion that was detected using MLPA in each patient were amplified in a single multiplex reaction. The multiplex reaction included the two controls of exon 3 of Myelin Protein Zero (MPZ3) and exon 18 of the MLH1 genes, as described by Di Bella et al. (2006) and Saugier-Veber et al., (2001) respectively. The relative peak areas for each tested exon were calculated as described above for MLPA.

\section{Breakpoint analysis}

Analysis was carried out on DNA samples from patients
F2, F48, F115, F118, P469, P259, P446, P291, P735, and $P 657$, all of which have a deletion of either exon 3 or exon 5. Results were used to verify the occurrence in our patients of the mutations Ex5del955 (systematic name g.50448_51402del955), Ex5del4232ins268 (systematic name g.47563_51794del4232g.56161_56430ins268), or Ex3del4765 (systematic name g.21560_26324del4765) previously detected in Czech patients by (Kozak et al. 2006), or mutations Ex3del6604ins8 (systematic name g.45041_51645delinsGGCACCTG) or Ex5del1881 (systematic name g.76772_78653del1881) previously detected in Spanish patients by Desviat et al. (2006). We designed primers (sequences available on request) to PCR amplify DNA fragments containing the reported breakpoints. A PCR amplification product of the expected size in our samples was considered diagnostic for the presence of the same breakpoints for these deletions. To verify that the absence of a PCR product was not due to an artifact, the D22S306 short tandem repeat locus was co-amplified in each reaction. Final confirmation of each breakpoint was based on sequence analysis.

\section{Acknowledgements}

This work was supported by the following fund of the Italian Ministry of Health: Ricerca corrente 2008 entitled "Le malattie genetiche con ritardo mentale". M.Vinci is a Ph. student of the "Dottorato di Ricerca in Genomica e Proteomica nella Ricerca Oncologica ed Endocrino-Metabolica" of the University of Palermo.

\section{References}

Akrami SM, Dunlop MG, Farrington SM, Frayling IM, MacDonald F, Harvey JF, Armour JA. Screening for exonic copy number mutations at MSH2 and MLH1 by MAPH. Fam Cancer 2005;4:145-9

Belanger-Quintana A, Garcia MJ, Castro M, Desviat LR, Perez B, Mejia B, Ugarte M, Martinez-Pardo M. Spanish tetrahydrobiopterin-responsive phenylalanine hydroxylase -deficient patients: evolution of seven patients on long-term treatment with tetrahydrobiopterin. Mol Genet Metab 2005; 86 Suppl 1: S61-6

Birk Moller L, Nygren AO, Scott P, Hougaard P, Bieber Nielsen J, Hartmann C, Guttler F, Tyfield L, Zschocke J. Low proportion of whole exon deletions causing phenylketonuria in Denmark and Germany. Hum Mutat 2007;28:207

Desviat LR, Perez B, Ugarte M. Identification of exonic deletions in the PAH gene causing phenylketonuria by MLPA analysis. Clin Chim Acta 2006;373:164-7

Di Bella MA, CaliF, Seidita G, Mirisola M, Ragusa A, Ragalmuto A, Galesi O, Elia M, Greco D, Zingale M, Gambino G, D'Anna RP, Regan R, Carbone MC, Gallo A, Romano V. Screening of subtelomeric rearrangements in autistic disorder: identification of a partial trisomy of 13q34 in a patient bearing a 13q;21p translocation. Am J Med Genet B Neuropsychiatr Genet 2006;5:584-90

Gable M, Williams M, Stephenson A, Okano Y, Ring S, Hurtubise M, Tyfield L. Comparative multiplex dosage 
analysis detects whole exon deletions at the phenylalanine hydroxylase locus. Hum Mutat 2003;21:379-86

Guldberg P, Rey F, Zschocke J, Romano V, Francois B, Michiels L, Ullrich K, Hoffmann GF, Burgard P, Schmidt H, Meli C, Riva E, Dianzani I, Ponzone A, Rey J, Guttler F. A European multicenter study of phenylalanine hydroxylase deficiency: classification of 105 mutations and a general system for genotype-based prediction of metabolic phenotype. Am J Hum Genet 1998;63:71-9

Kozak L, Hrabincova E, Kintr J, Horky O, Zapletalova P, Blahakova I, Mejstrik P, Prochazkova D. Identification and characterization of large deletions in the phenylalanine hydroxylase (PAH) gene by MLPA: evidence for both homologous and non-homologous mechanisms of rearrangement. Mol Genet Metab 2006;89:300-9

Lee YW, Lee DH, Kim ND, Lee ST, Ahn JY, Choi TY, Lee YK, $\mathrm{Kim} \mathrm{SH}, \mathrm{Kim}$ JW, Ki CS. Mutation analysis of PAH gene and characterization of a recurrent deletion mutation in Korean patients with phenylketonuria. Exp Mol Med 2008;40:533-40

Mirisola MG, Cali F, Gloria A, Schinocca P, D'Amato M, Cassara G, Leo GD, Palillo L, Meli C, Romano V. PAH gene mutations in the Sicilian population: association with minihaplotypes and expression analysis. Mol Genet Metab 2001;74:353-61
Romano V, Dianzani I, Ponzone A, Zammarchi E, Eisensmith R, Ceratto N, Bosco P, Indelicato A. Prenatal diagnosis by minisatellite analysis in Italian families with phenylketonuria. Prenat Diagn 1994;14:959-62

Saugier-Veber P, Drouot N, Lefebvre S, Charbonnier F, Vial E, Munnich A, Frébourg T. Detection of heterozygous SMN1 deletions in SMA families using a simple fluorescent multiplex PCR method. J Med Genet 2001;38:240-3

Schouten JP, McElgunn CJ, Waaijer R, Zwijnenburg D, Diepvens F, Pals $G$. Relative quantification of 40 nucleic acid sequences by multiplex ligation-dependent probe amplification. Nucleic Acids Res 2002;30:e57

Scriver CR, Kaufman S. Hyperphenylalaninemia: phenylalanine hydroxylase deficiency. In The Metabolic and Molecular Bases of Inherited Disease (Scriver CR, Beaudet AL, Valle D, Sly WS, Childs B, Volgestein B, eds), 2001, 1667-724, McGraw-Hill, New York, NY

Zeng F, Ren ZR, Huang SZ, Kalf M, Mommersteeg M, Smit M, White S, Jin CL, Xu M, Zhou DW, Yan JB, Chen MJ, van Beuningen R, Huang SZ, den Dunnen J, Zeng YT, Wu Y. Array-MLPA: comprehensive detection of deletions and duplications and its application to DMD patients. Hum Mutat 2008;29:190-7 